\title{
Characterization of optical spectrum in laser beam welding of dissimilar aluminum-copper joints and time- dependent correlation to process stages
}

\section{Marc Seibold, Klaus Schricker, Jean Pierre Bergmann}

Marc Seibold, Klaus Schricker, Jean Pierre Bergmann, "Characterization of optical spectrum in laser beam welding of dissimilar aluminum-copper joints and time-dependent correlation to process stages," Proc. SPIE 11144, Photonics and Education in Measurement Science 2019, 111440 (17 September 2019); doi: 10.1117/12.2533476

SPIE Event: Joint TC1 - TC2 International Symposium on Photonics and Education in Measurement Science 2019, 2019, Jena, Germany 


\title{
Characterization of Optical Spectrum in Laser Beam Welding of Dissimilar Aluminum-Copper Joints and Time-Dependent Correlation to Process Stages
}

\author{
Marc Seibold*a, Klaus Schricker ${ }^{\mathrm{a}}$, Jean Pierre Bergmann ${ }^{\mathrm{a}}$ \\ ${ }^{a}$ Technische Universität Ilmenau, Production Technology Group, Gustav-Kirchhoff-Platz 2, 98693 Ilmenau, \\ Germany
}

\begin{abstract}
Electromobility has been a strongly growing market for years. This is the reason for the demand for battery technology and electric components continue to increase. In these technologies, the material copper is indispensable due to its high electrical conductivity. With the same electrical conductivity, substituting aluminum to copper leads to a reduction in costs and weight. For example, substitution is not possible at connecting points, therefore dissimilar aluminum-copper joints are highly important. In micro processing, pulsed laser beam welding is applied to achieve a slender weld seam. However, the mixing of both joining partners leads to the formation of intermetallic phases during welding. This requires a precise detection of the process stage in order to limit the weld seam depth close to the interface between both materials.

In this paper, a pulsed laser welding process between aluminum and copper was tested by using a fiber laser (IPG-YLM$450 / 4500-\mathrm{QCW}$, pulse duration $<10 \mathrm{~ms}$ ). The optical spectrum of the welding process was detected by spectrometers in the visible light range. When aluminum is welded with copper, the wavelength spectrum changes due to the material dependent emission. The maxima within the wavelengths of each joining partner could thus be determined and transferred to photodiodes with suitable bandpass filters. This leads to an increase of the temporal resolution during the measurement compared to spectrometers, allowing the analyzation of the time-related signal characteristics. A difference between heat conduction welding and deep welding as well as the transition from upper to lower sheet metal could be determined.
\end{abstract}

Keywords: laser beam welding, aluminum-copper joints, process emission, photodiodes, spectrometer, deep penetration welding, dissimilar joints

\section{INTRODUCTION}

While the electromobility sector is growing, the demand for weight savings through heavy components, especially the battery, is increasing. Based on this sustained trend of lightweight construction, a reduction in weight can be achieved by replacing copper with aluminum for electrical applications. To achieve the same specific electrical conductivity, aluminum $\left(\gamma\left(20^{\circ} \mathrm{C}\right)=34 \mathrm{MS} / \mathrm{m}\right)$ requires four times the cross-section of copper $\left(\gamma\left(20^{\circ} \mathrm{C}\right)=57 \mathrm{MS} / \mathrm{m}\right)$. With a density of $2.7 \mathrm{~kg} / \mathrm{m}^{3}$ of aluminum compared to $8.93 \mathrm{~kg} / \mathrm{m}^{3}$ of copper, the mass can be reduced by up to $50 \%$ and the costs up to $85 \%$ even if the cross-section is enlarged [1 - 4], concerning the fluctuation in copper prices. Although these arguments are advantageous for replacing copper with aluminum, copper cannot be completely replaced. For example, in connectors or due to technological requirements in batteries, copper is not always interchangeable, which is the reason for the need of copper-aluminum mixed connections at these junctions. However, the manufacturing of such dissimilar joints is associated with major challenges. Based on the two-phase diagrams of aluminum-copper, it can be seen that such aluminum-copper joints tend to form strong intermetallic phases. These intermetallic phases are characterized by high brittleness and poor electrical conductivity [5], which is the reason why they should be minimized [6]. Solid-phase joining processes are therefore predestined for this, as a melt pool between the two materials can be avoided and phase formation can thus be strongly limited, as shown for ultrasonic welding [7] or friction stir welding [6]. However, due to the high process forces, these processes can only be used to a limited extent for applications in micro joining. In general, 
pulsed laser beam processes are very well suited for this purpose, although a molten bath between aluminum and copper cannot be avoided in such processes. The molten bath has a very high dynamic behavior, which leads to a high degree of mixing of both materials and to the strong formation of intermetallic phases. A limitation of intermetallic phases can be achieved by limiting the mixing of both materials.

In order to meet this challenge, aluminum-copper joints are produced in overlap configuration, using aluminum as the upper joining partner and copper as the lower joining partner. This configuration is chosen based on the solubility up to the formation of intermetallic phases and on the other hand the higher absorption of $4 \%$ for aluminum and $1 \%$ for copper $[8,9]$. The solubility of copper in aluminum is less than $10 \%$ by mass., whereas the solubility of aluminum in copper is more than $30 \%$ by mass. [6]. This results in a larger tolerance range for the amount of molten copper. In addition, intermetallic phases with a higher percentage of copper have a greater hardness and a higher electrical resistance [5].

This requires an exact knowledge of the welding depth. In general, the use of an optical coherence tomography (OCT) system is recommended for determining the welding depth during the process. However, the transition from aluminum to copper is the decisive factor which, however, cannot be detected by OCT. For the determination of the material transition, the emission of the metal and metal vapor during welding can be utilized [10]. Because the measuring principle requires evaporation of the second joining partner, mixing cannot be avoided, but minimized. Due to the material-dependent emission, the change from aluminum to copper can be detected due to changes in the spectrum [12]. A schematic of the process is given in Fig. 1. After switching on the laser beam, heat conduction welding takes place first (1), before the capillary forms after a very short time (2). Then the welding process reaches the interface between the two materials (3) which is why emissions of copper will also be available from this point in time. Due to the unstable process conditions when welding into aluminum, this time cannot be predicted exactly. At this point, the process has to be stopped in order to avoid further mixing of the materials (4).

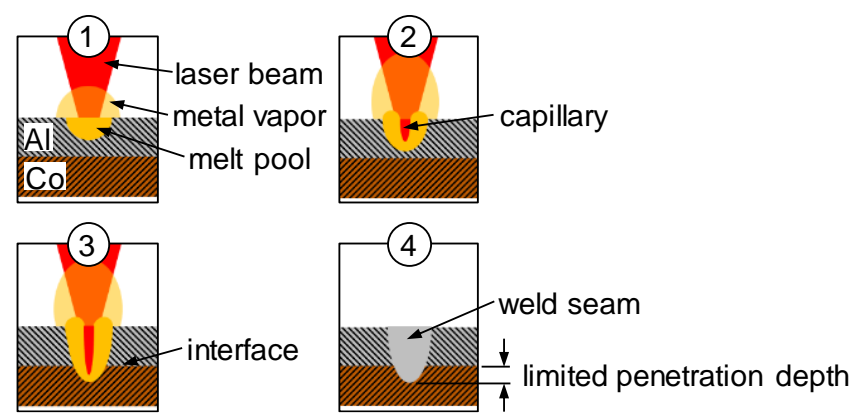

Fig. 1 Different process stages in welding aluminum-copper joints

In this paper, spectrometer measurements are used to record the process emissions and to assign characteristic wavelengths to the different process stages (1-3) in pulsed laser beam welding. Through the further use of photodiodes, the signals can be resolved in time to capture different process phases even for pulses $<10 \mathrm{~ms}$. Accompanying highspeed recordings to support the investigations and increase process understanding. The investigations thus creates the starting point for further implementation of a process control in subsequent investigations.

\section{EXPERIMENTAL SETUP}

As shown in Fig. 1, the following measurement systems are used to determine the emission spectra. The aluminum alloy EN AW-1050A and the oxygen-containing copper Cu-ETP are used as sample materials. The sample size with $\mathrm{L}=50$ $\mathrm{mm}, \mathrm{B}=30 \mathrm{~mm}$ and $\mathrm{H}=0.5 \mathrm{~mm}$ is chosen so that heat accumulation can be eliminated due to a too small edge distance. A clamping device ensures the position as well as the contact between the specimens. The fiber laser IPG YLR$450 / 4500-\mathrm{QCW}$ is used as laser source. This Q-switched laser has a maximum pulse energy of $45 \mathrm{~J}$ and a maximum pulse duration of $t_{p}=50 \mathrm{~ms}$. The focused spot has a diameter of $d_{f}=176 \mu \mathrm{m}$ and can apply a maximum pulse power of $\mathrm{P}_{\max }=4500 \mathrm{~W}$. Due to the control times of the power supplies of $10 \mu \mathrm{s}$, the laser beam source is suitable for the implementation of process control. This beam source emits at $1070 \mathrm{~nm}$ and is therefore outside the visible wavelength spectrum. In order to obtain an almost unidirectional emission, single pulse spot welds are performed. The only exception is the illustration of the initial challenge in chapter 3.1, for which a continuous weld seam was produced. A 
pulse ramp of 0 to $1200 \mathrm{~W}$ with a duration of $8 \mathrm{~ms}$ is used for all welds. This makes it possible to display changes during the welding process in a larger time interval. The process emission to be determined lies in the optically visible range, which is why there is no overlap with the laser wavelength. For the determination of the material-specific emission spectra, the VIS spectrometer AvaSpec-ULS2048CL-EVO is used. Because of the high process emission, a low exposure time of $0.1 \mathrm{~ms}$ is selected. The spectrometer measurements with a frequency of $370 \mathrm{~Hz}$, which results in two measurements per pulse at the selected pulse duration. The high-speed camera Photron Fastcam SA-X2 with a Navitar $12 \mathrm{x}$ Zoom objective is used to validate the measurements. By using a 1064-40 nm notch filter, process emissions in the visible range can be detected and the laser wavelength blocked at the same time. The high-speed camera is positioned at an angle of $15^{\circ}$ to the optical axis. This allows an almost coaxial recording. In addition, a crossjet was used to protect the laser optic and the bandpass filter against spatters and metal vapor. The crossjet was placed $50 \mathrm{~mm}$ above the top of the sample and is thus below all measuring instruments.

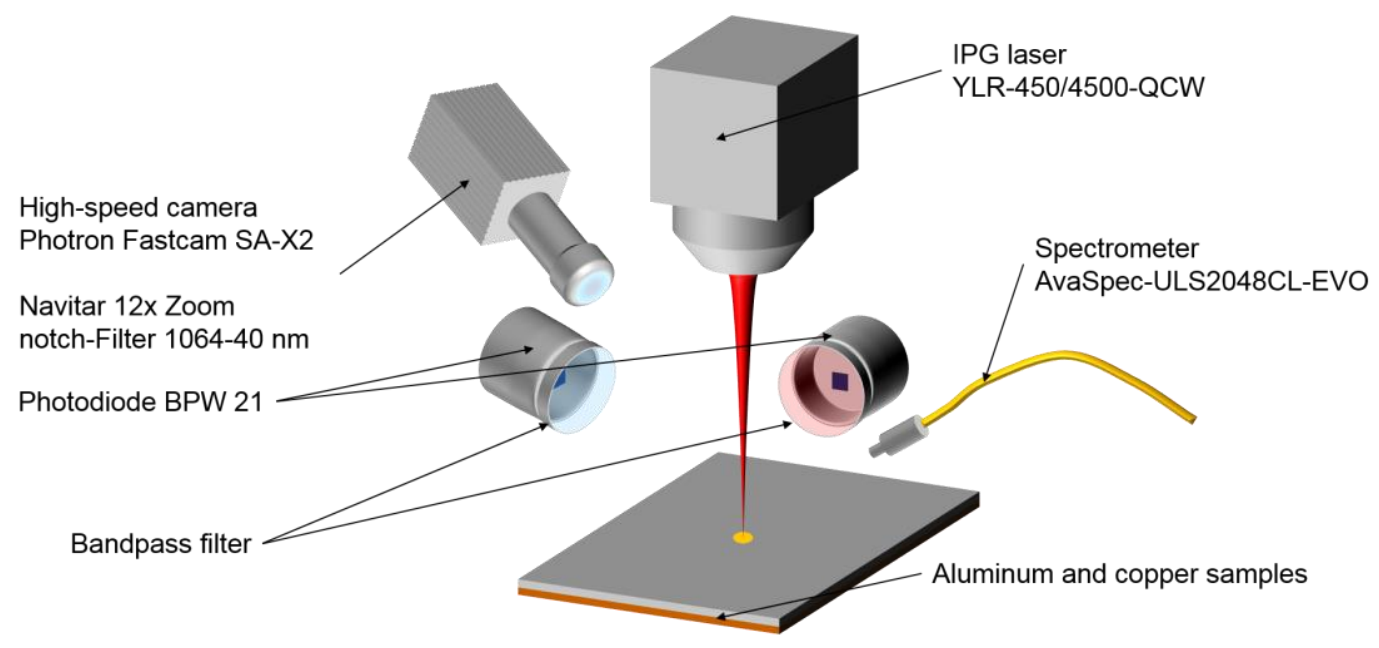

Fig. 2 experimental setup with a schematic view of all used components

After determining the material-dependent spectra, photodiodes are directed at the process with the respective bandpass filters. The angle between the photodiodes and the optical axis of the laser beam is less than $15^{\circ}$ to capture the emission directly in the keyhole. The photodiode signals were recorded by a Dewetron DEWE-5000 measuring device.

\section{RESULTS AND DISCUSSION}

\subsection{Characterization of the laser process}

The main challenge in pulsed-laser beam welding of aluminum and copper, a longitudinal is given in Fig. 3, which shows a continuous weld between both materials. In the upper area the solidified aluminum is to be seen and in the lower area the bonded copper. The single pulses of the weld overlap in such a way that a common molten pool is formed. A distinctive point is the connection between aluminum and copper. It can be seen that, despite identical pulses, changes in the welding depth in copper can be detected on the one hand and on the other hand, not continuously welded into copper. 


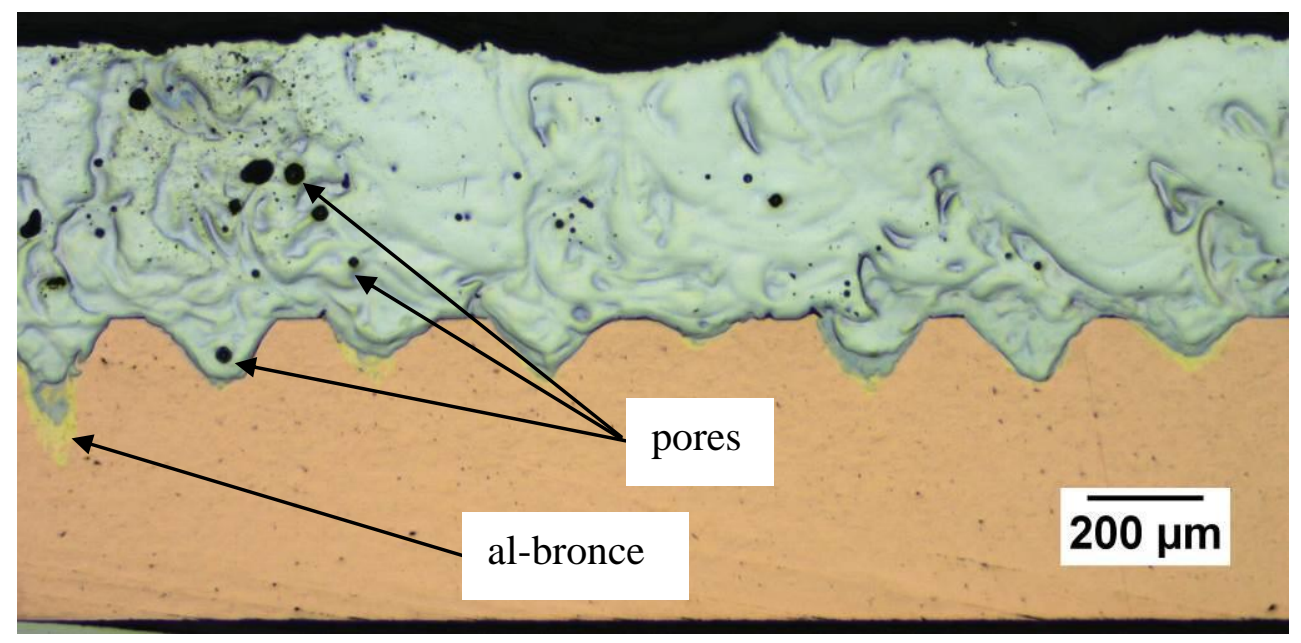

Fig. 3 longitudinal section of a continuous laser welding. $P_{\max }=1800 \mathrm{~W}, \mathrm{~d}_{\mathrm{f}}=176 \mu \mathrm{m}, \mathrm{v}=3$ $\mathrm{m} / \mathrm{min}, \mathrm{t}_{\mathrm{P}}=8 \mathrm{~ms}$

From the cross section (Fig. 4) of a spot weld, it can be seen that the appearance of the weld seam is comparable. In the upper area the wide, solidified aluminum melting bath is visible. The collapsed upper side of the seam is typical for a deep welding process and results from the molten metal flowing to the position of the vapor capillary after the end of the process. The width of the aluminum melt pool changes into a very small melt pool within the copper sheet.

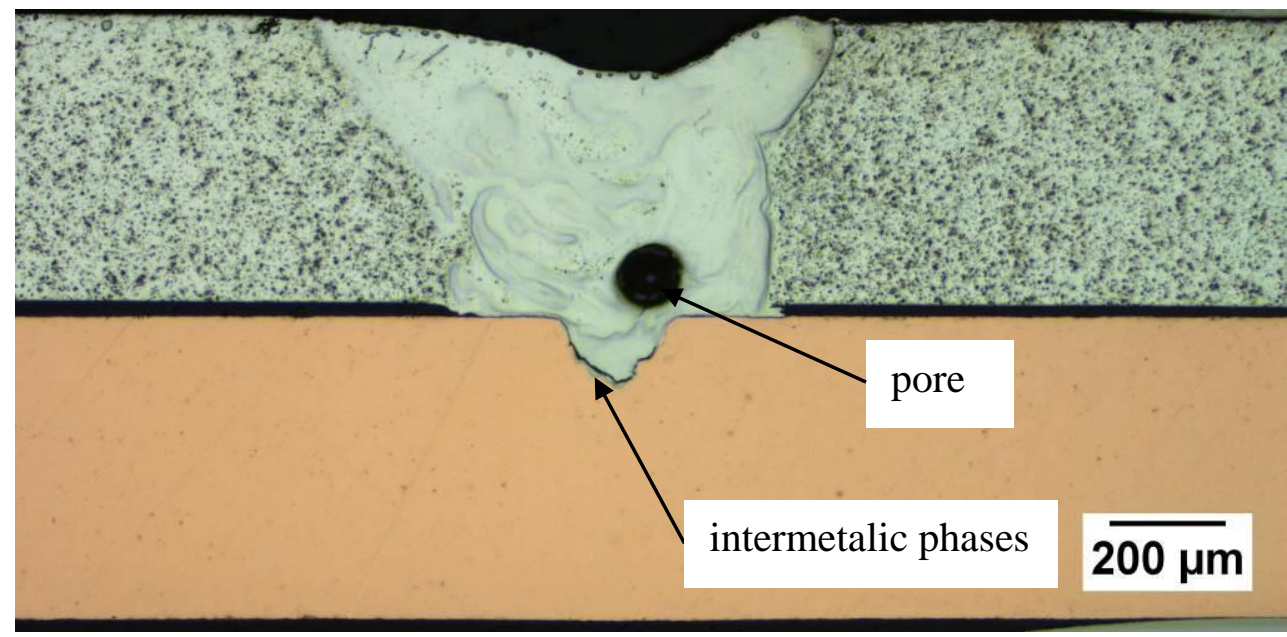

Fig. 4: cross section of a spot weld from aluminum to copper. $\mathrm{P}_{\max }=1800 \mathrm{~W}, \mathrm{~d}_{\mathrm{f}}=176 \mu \mathrm{m}$, $\mathrm{v}=3 \mathrm{~m} / \mathrm{min}, \mathrm{t}_{\mathrm{P}}=8 \mathrm{~ms}$

As already mentioned in the introduction, the process goes through different phases during laser welding. Fig. 5 shows the different phases during the welding process by high speed images (a-d). The images e) and f) show the process emission after the pulse was over. The first two images a) and b) show the area of heat conduction welding. What can be seen very well, is that the process emission of aluminum is partly from the molten bath and partly from the metal vapor. The emission of the metal vapor is so intense that it is reflected back from the sheet surface. This can also be seen in images c) and d), which show the deep welding process. Due to the steep angle of attack of $15^{\circ}$ the capillary is visible in the middle of the images. This shows, that during this phase, material can rise from the lower copper sheet to the vapor capillary. Around the keyhole a symmetric molten bath can be seen. On this there is a layer of aluminum oxide. This is in solid form due to the high melting temperature. Due to the flow and the molten pool rising from the sheet level, the aluminum oxide layer is pushed radially out of the middle. On the edge of the aluminum oxide layer directed towards the 
capillary, bright dots can be seen in the high-speed images c) and d), which indicate that the oxide is evaporating. During the deep welding process, spatters may be detached. These are molten at the time of separation and also emit radiation, which is clearly visible in Figure c). Around this splash a layer of evaporating material is formed, which also adds to the emission. After the end of the pulse, the still molten material continues to glow for a while, which can be seen in the high-speed images e) and f). With increasing time between the ends of the laser pulse, the process light continues to decrease.
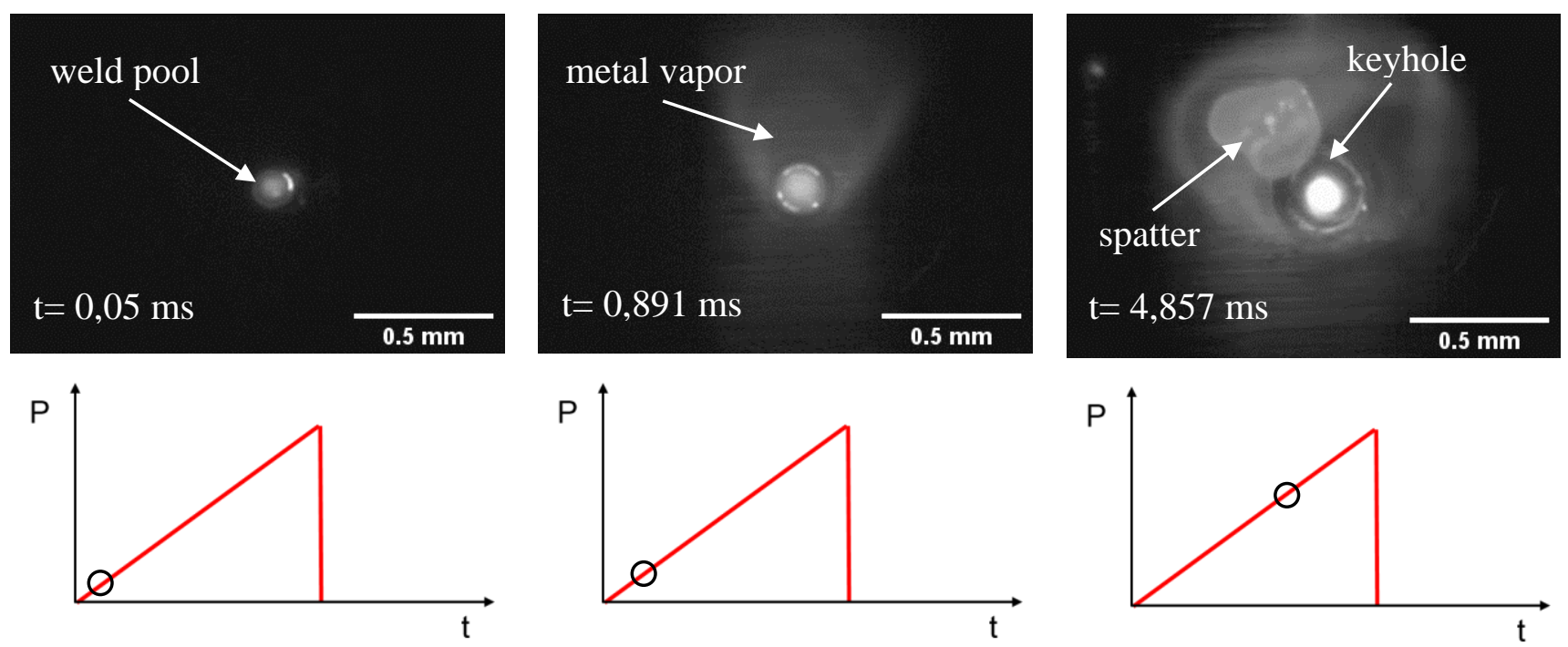

a)

b)
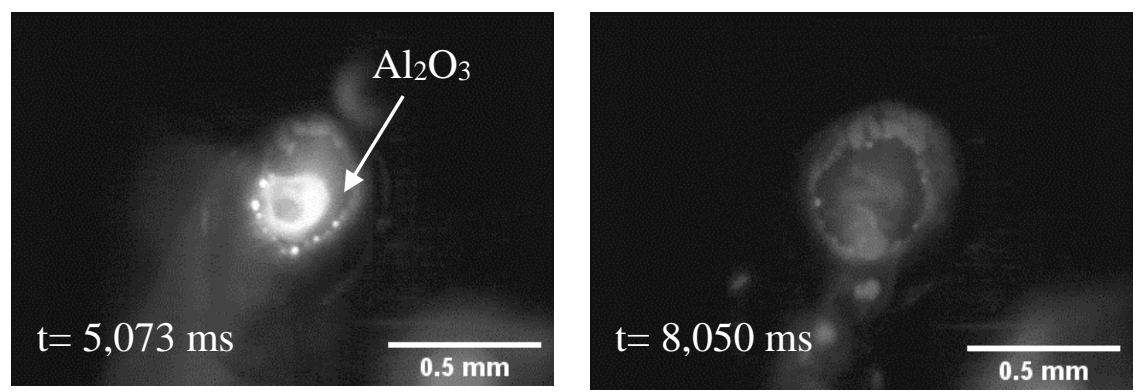

c)
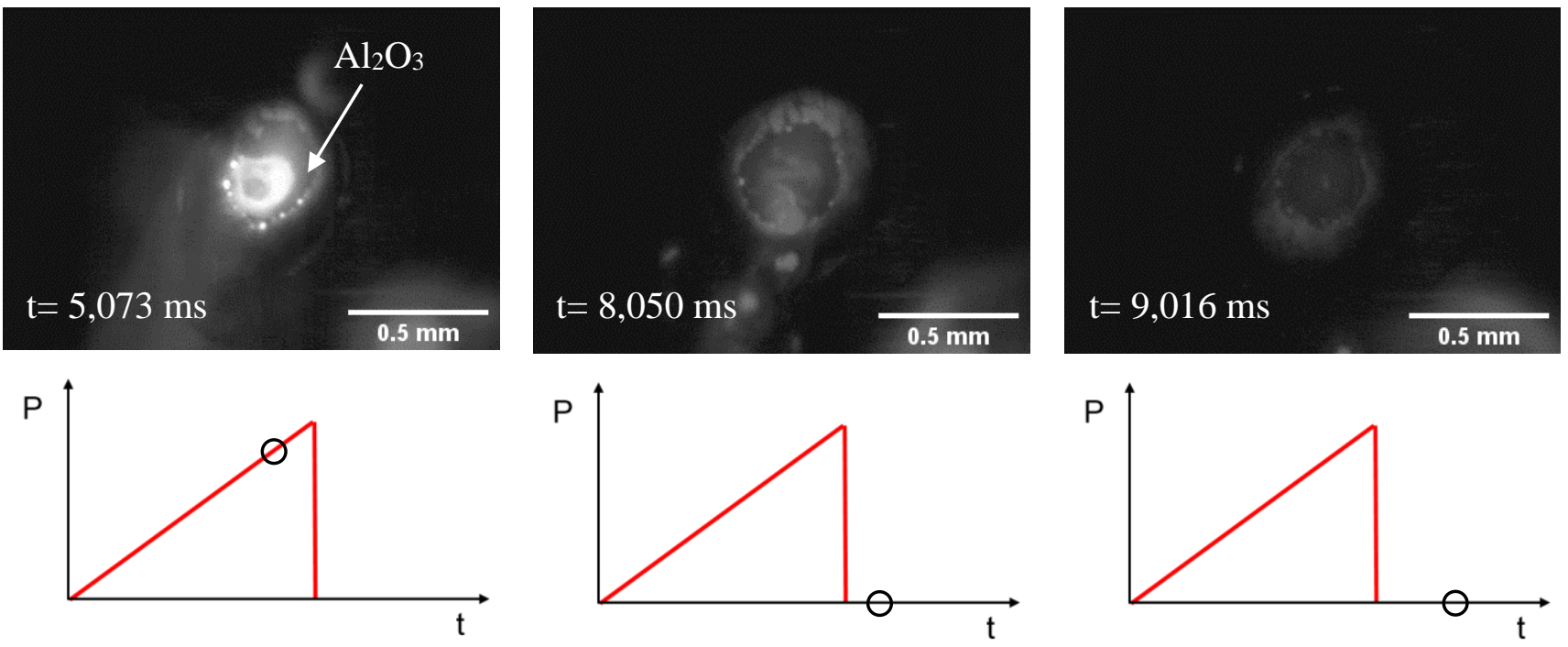

d)

e)

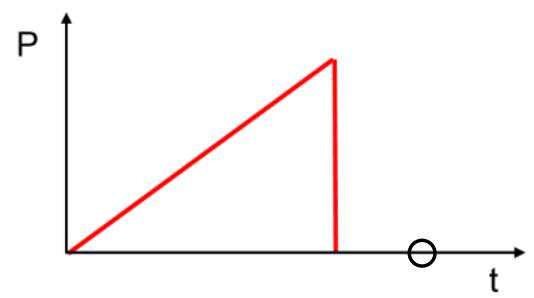

f)

Fig. 5 Different shots of the high-speed camera during a) - d) and after the process e) $-\mathrm{f}$ )

\subsection{Identification of characteristic wavelengths in emission spectra}

In order to exclude the influence of ambient light in the process signal, a spectrometer measurement without the process is recorded and subtracted from the measurements with a process. Fig. 6 shows such a difference signal when aluminum is welded into copper. On the horizontal axis is the wavelength from $300 \mathrm{~nm}$ to $900 \mathrm{~nm}$, which covers a range from ultraviolet to infrared. The vertical axis shows the intensity of the measured light within the exposure time. It can be seen 
that the welding process emits radiation over the visible wavelength range. The basic emission is surpassed by narrowband peaks, which can be derived from the emission of the ionization stage. The first striking peak almost combines the two most energetic emissions of aluminum, at $394.4 \mathrm{~nm}$ and $396.15 \mathrm{~nm}$ together [12]. In particular, the two large peaks at $470 \mathrm{~nm}$ and $485 \mathrm{~nm}$ can be explained by the evaporation of aluminum oxide. The last peak marked represents the emission of copper, because the evaporating material is transported out of the interface through the capillary, which is why the peak is significantly lower [11]. Due to the short exposure time of $0.1 \mathrm{~ms}$, the spectrometer only records a short time frame of the welding process and thus provides limited informative value with regard to time-dependent process recording. For this reason, the measured intensity of each weld is slightly different, but the characteristic peaks are reproducible for all recorded spectra.

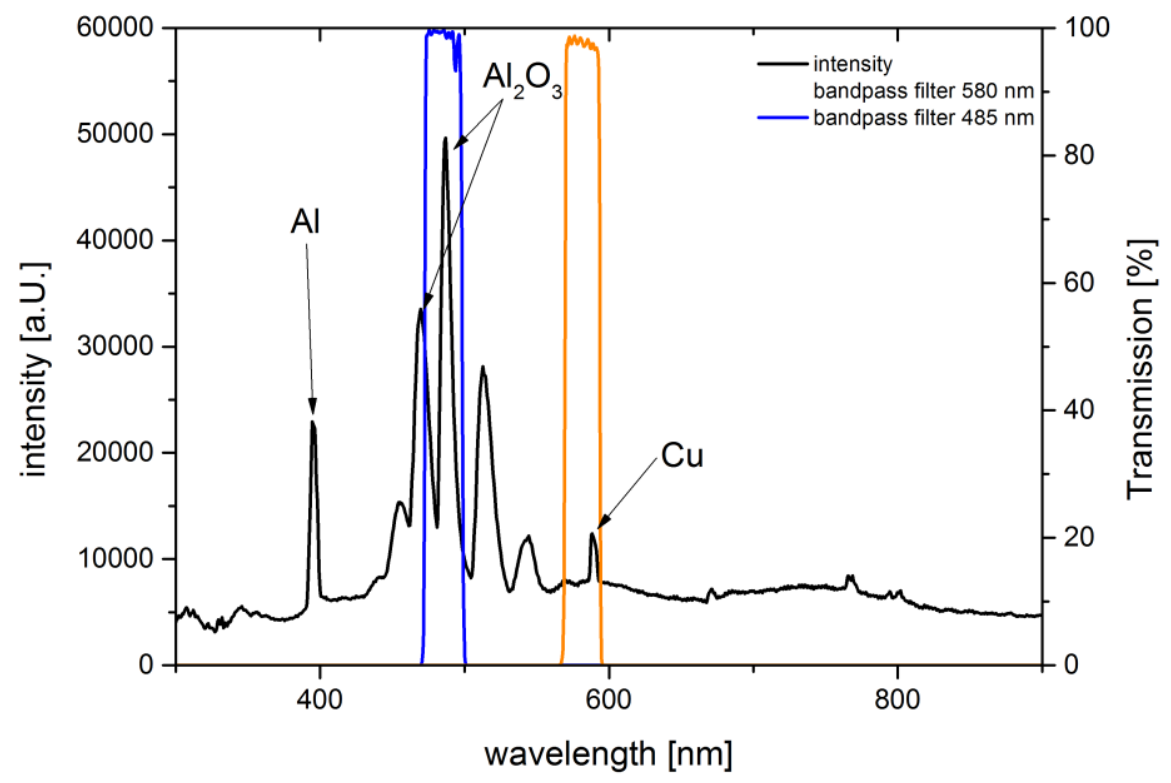

Fig. 6 Spectrometer differential signal of an aluminum-copper weld with characteristic wavelengths for $\mathrm{Al}, \mathrm{Al}_{2} \mathrm{O}_{3}$ and $\mathrm{Cu}$ following [11].

In order to achieve higher sampling rates, photodiodes with bandpass filters were integrated into the experimental setup. The bandpass filters are selected as a result of the spectrometer signals. For the detection of copper, a filter with $580 \mathrm{~nm}$ and a half width of $20 \mathrm{~nm}$ is used. For the determination of the aluminum radiation, the emission at $394.4 \mathrm{~nm}$ is not taken into account, but the emission of the aluminum oxide which offers advantages due to the detection of the interface. The bandpass filter used transmits at a wavelength of $485 \mathrm{~nm}$ with a half width of $20 \mathrm{~nm}$. This relation between bandpass filter and spectrometer signal is also shown Fig. 6. On the right vertical axis, the transmission of the bandpass filters is plotted in percent. The transmission data were provided by the data sheet [13]. The range of maximum transmission of the bandpass filters overlap with the material-dependent peaks.

\subsection{Time-dependent progression of emission wavelength $485 \mathrm{~nm}$ and $580 \mathrm{~nm}$}

The photodiodes of type BPW 21 allow a significantly higher recording rate, which can achieve up to $100 \mathrm{kHz}$. This allows the time-dependent evaluation of the process based on the emissions at the characteristic wavelengths as shown in Fig. 7. Thereby, the voltage of the photodiodes is plotted over time in milliseconds. The measurement starts $5 \mathrm{~ms}$ before the welding process. In order to eliminate the influence of the surrounding light during photodiode measurements, the voltage signal is set to zero before the measurement. The blue curve shows the signal of the photodiode equipped with the $485 \mathrm{~nm}$ bandpass filter, which represents the aluminum. The second voltage signal represents copper. The higher emission of $\mathrm{Al}_{2} \mathrm{O}_{3}$ compared to the copper peak leads to much higher voltages compared to the second signal (see also chapter 3.2). Additionally, the photodiode is highly sensitive in this wavelength range [14]. With the start of the welding 
process, the emission increases abruptly. After a short period at a relatively constant level, the signal of the $485 \mathrm{~nm}$ photodiode increases again. This peak corresponds to the evaporation of the aluminum oxide layer on top. This point in time is marked with the first dotted lines. The second aluminum oxide layer, which acts as a separating layer between aluminum and copper, evaporates shortly afterwards which is represented by the second dotted line. This can be seen from the second peak of the $485 \mathrm{~nm}$ photodiode. Almost immediately afterwards, a peak form in the second photodiode signal. This can be explained by the melting and evaporation of copper. After the peaks, the photodiodes assume an approximately constant voltage signal. Despite increasing power, due to the power ramp, the photodiode signal does not increase further. From the general law that the sum of absorption and transmission is equal to the emission, an increase in power should lead to an increase in emission. This means that the input power must be removed from the sample plate via other options. After the pulse, the photodiode signals do not decrease so abruptly, but take on a logarithmic decay. This can be explained by the residual radiation of the solidifying melt. This effect is also recognizable in the high-speed images Fig. 5.

It should be noted that the material-dependent peaks cannot be noticed within the high-speed recordings. The reason for this is the wavelength band transmitted by the notch filter. Due to the small wavelength peaks, these are not noticeable in the general emission. The left picture shows a snapshot of the heat conduction welding before coupling into the aluminum sheet. The second image of the high-speed camera shows the welding during the deep welding phase. Due to the irregular evaporation of this oxide layer, the photodiode signal with the $485 \mathrm{~nm}$ bandpass filter varies more in the deep welding process (2) than in the heat conduction process (1).
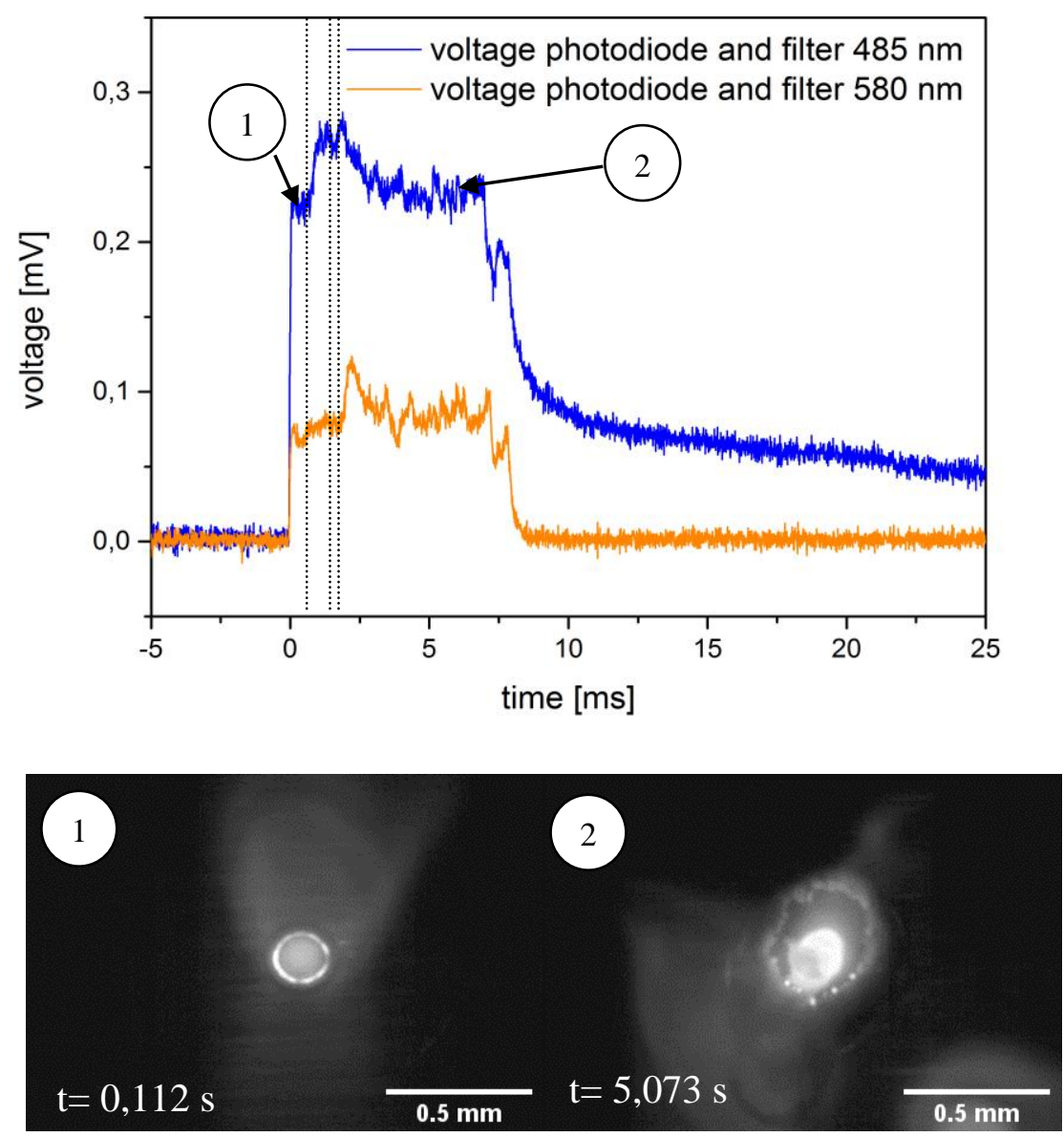

Fig. 7 Representation of a photodiode signal of an aluminum copper weld with two sample images of a high-speed video Timestamp and scale are missing in videos 
Fig. 8 illustrates the necessity of a time-dependent recording of the process. Due to the instabilities in the welding process, the interface for each pulse is reached after a slightly different time. Three photodiode signals are plotted on top of each other. Each of the three signal groups is $0 \mathrm{~ms}$ at $0 \mathrm{mV}$ and is plotted on top of each other for better comparability. It is well recognizable, that with three identical welds the photo diode signals differ clearly. The time, it takes for the welding to be represented by the aluminum oxide peaks, varies. The sequence of the peaks is identical for all three welds. First there are two closely consecutive aluminum oxide peaks (1) (2), followed by one copper peak (3). All welds reach the welding phase in copper at different times. From this time on, there is a common molten pool between aluminum and copper. With further energy input, this leads to the further melting of copper, which is why the molten pool becomes richer in copper. As mentioned at the beginning, copper has only a limited solubility in aluminum and leads to the formation of intermetallic phases if the solubility is exceeded. Based on the time-dependent detection of the interface, a novel process control will be developed in the future.

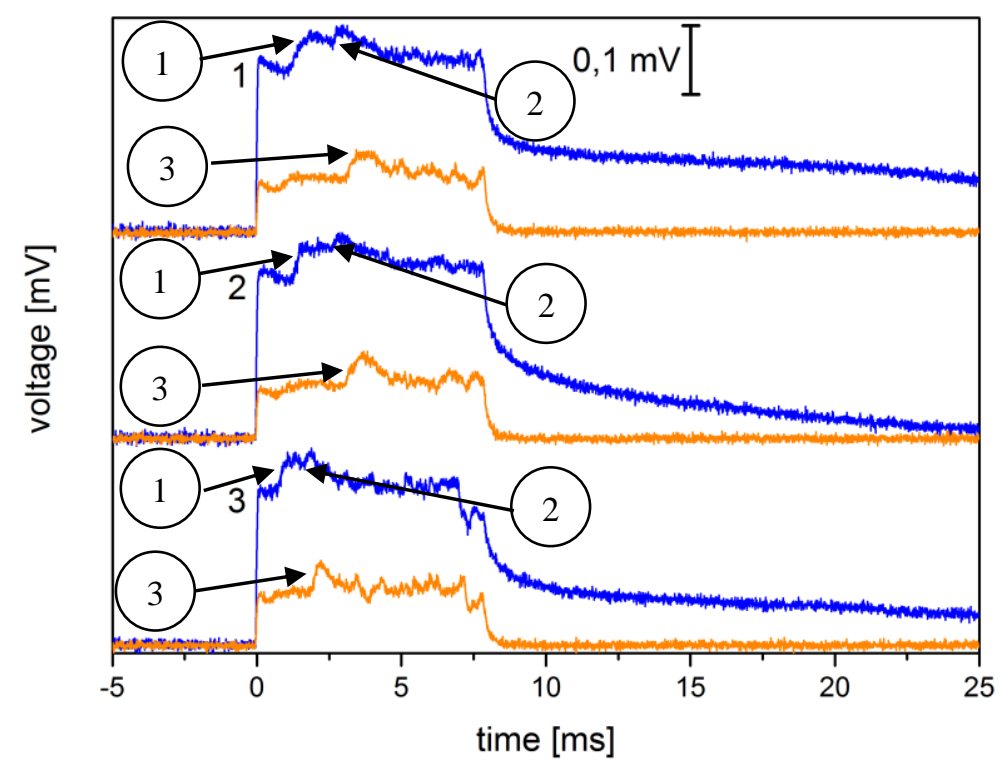

Fig. 8 Comparison of three photodiode signals

Fig. 9 shows the comparison of two welding processes with different long pulses. The measured values shown in blue and orange correspond to the welding with a pulse length of $8 \mathrm{~ms}$. The other two measured values correspond to a weld with a pulse length of $4 \mathrm{~ms}$. The difference between the pulse shapes is not only the pulse duration but also the time of welding into the copper sheet. It can be seen that at a pulse duration of $4 \mathrm{~ms}$, the welding takes place at about $50 \%$ of the pulse duration. This time is only minimally earlier than with a pulse duration of $8 \mathrm{~ms}$. Furthermore, due to the faster increase in power, the separated aluminum oxide peaks almost unite. 


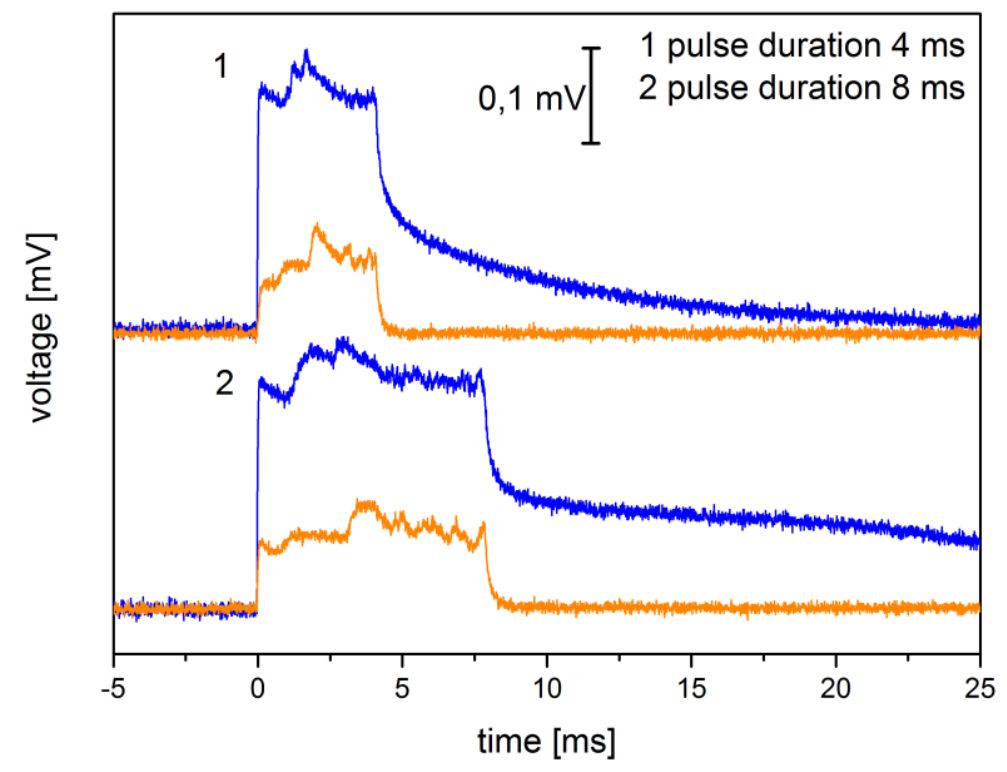

Fig. 9 Comparison of different welding with a) Ramp of $8 \mathrm{~ms}$ and b) a Ramp of $4 \mathrm{~ms}$

Fig. 10 shows the difference between the voltage signals as a function of the distance to the process zone. On the horizontal axis the distance of the photodiode to the process is plotted. The vertical axis corresponds to the measured voltage signal of the photodiodes. For better comparability, only the diode signals equipped with a bandpass filter of 485 $\mathrm{nm}$ were used. From each of the 5 identical tests, the maximum voltage value was recorded and represents the mean value. The error bars correspond to the maximum measured deviation of the mean values. What can be seen very well from this figure is that the distance between the photodiodes and the process zones has a minimal influence. Especially at distances greater than $120 \mathrm{~mm}$ the voltage signal is constant. At distances smaller than $120 \mathrm{~mm}$, a higher voltage can be detected. However, this distance can cause damage to the photodiodes due to splashes and metal vapor, these are not process relevant. With this knowledge, the working range could be selected in such a way that the influence of the distance could be omitted. 


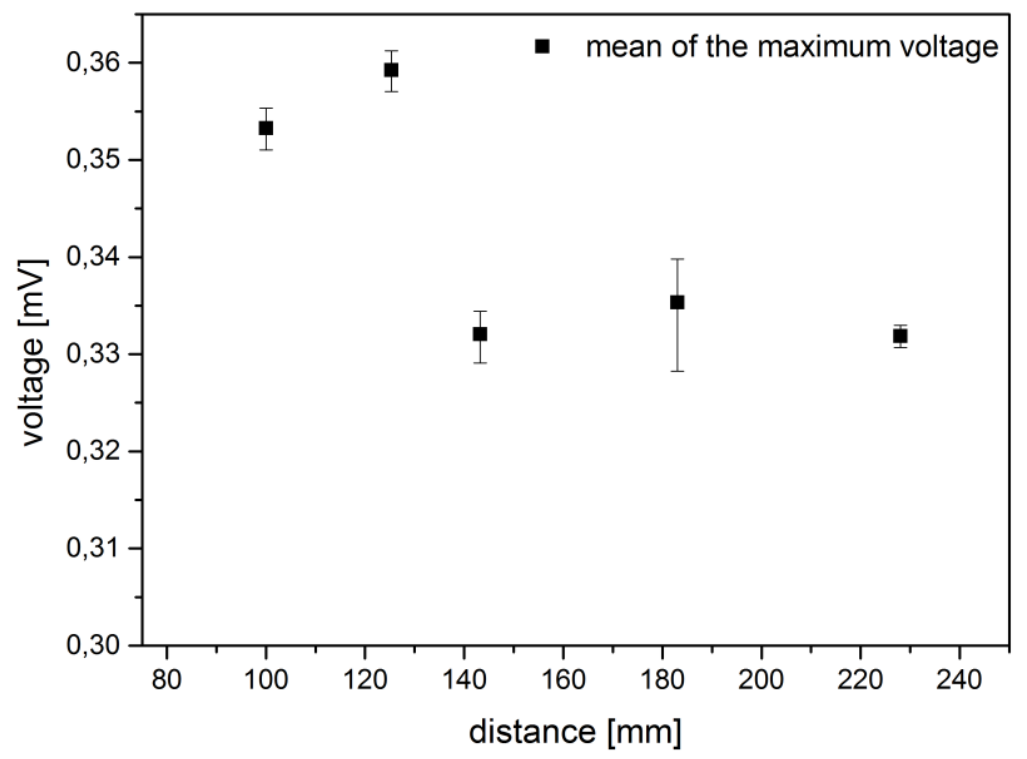

Fig. 10: measurements of photo diode signals with different distance between diodes and process area.

In addition to the distance, the angle between the photodiodes and the sample surface plays a decisive role. This angle will be indicated between the variations to the optical axis. This means that a coaxial image takes the angle $0^{\circ}$ and an image parallel to the sheet surface takes the angle $90^{\circ}$. The Fig. 11 represents three different diode signals at different angles. The entire process is not displayed, but only the initial ramp. The recognizable phenomena are, on the one hand, that the signal increase is smaller with increasing angle and, on the other hand, a plateau develops as a result. Due to the fact that less process emission from the capillary hits the photodiode at a flat angle, the photodiode only records the emission of the metal vapor at the flat angle. The resulting metal vapor initially increases constantly as the process progresses. This increase in metal vapor is also clearly visible in Fig. 5 b) -d). 


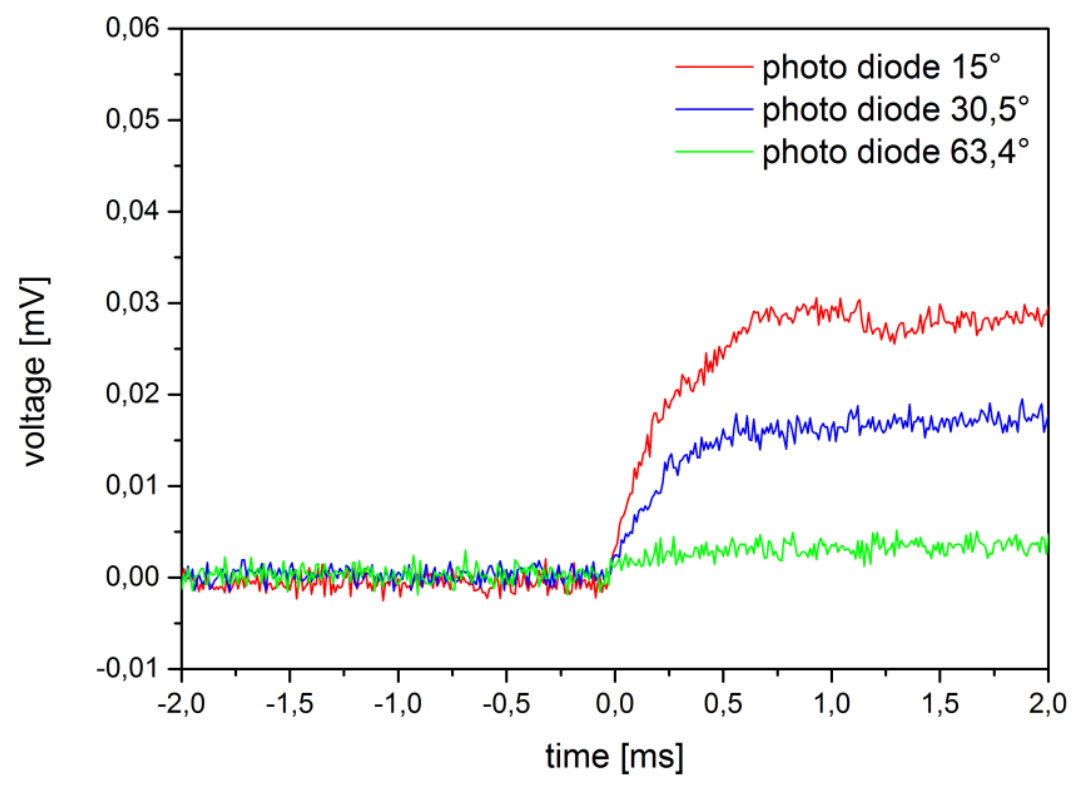

Fig. 11 Different photodiode signal increases depending on the angle to the optical axis.

\section{CONCLUSION}

In this paper it was shown that the process emission of a laser beam welding of aluminum and copper can be measured. Despite the overlap configuration, it can be seen that the emission of the lower sample material, copper, can be detected. The evaporating material can leave the joining zone via the capillary. This circumstance could be shown by measurements of a spectrometer. In the spectrometer signal, emissions of aluminum, aluminum oxide and copper can be detected. Because the measuring rate of the spectrometer is limited to $370 \mathrm{~Hz}$, the temporal signal curve cannot be detected accurately. The use of BPW 21 photodiodes and bandpass filters for the material-specific emission made it possible to subdivide the welding process. It was shown that the through-welding of the aluminum oxide layers as well as the copper sheet surface is imaged in the photodiode signals. The passage through a layer can be seen as a short-term increase of the diode voltage. The comparison with the high-speed camera images shows the difference between heat conduction welding and deep penetration welding. Due to the irregular evaporation of the aluminum oxide during the deep welding process, the fluctuating process signal can be recognized.

\section{ACKNOWLEDGEMENTS}

We thank the BMWi within the Zentrales Innovationsprogramm Mittelstand (ZIM) for funding the project. (FKZ 16KN053046) 


\section{REFERENCES}

[1] Aurubis Stolberg "Material Datasheet Cu-DHP" < http://www.aurubisstolberg.com/wdb/band/eng/Copper/Cu-DHP-PNA\%20219_EN.pdf > (21.05.2019)

[2] Aurubis Stolberg "Material Datasheet Cu-ETP" < http://www.aurubisstolberg.com/wdb/band/eng/Copper/Cu-ETP-PNA\%20211_EN.pdf > (21.05.2019)

[3] AlCuMet "EN AW-1050A" < https://www.alcumet.de/daten/Datenblatt\%20\%20A199,5\%20-\%20EN\%20AW-1050A.pdf > (02.05.2019)

[4] Alcunnect "Comparison of Aluminium and Copper" < https://www.alcunnect.de/VergleichAluminium-und-Kupfer?language_id=3 > (15.05.2019)

[5] Schmalen, P.,Plapper, P. "Evaluation of Laser Braze-welded Dissimilar Al-Cu Joints, Physics Procedia, 506 - 514, (2016)

[6] Bergmann, J. P.,Petzoldt, F.,Schürer, R.,Schneider, S. "Solid-state welding of aluminum to copper-case studies, Welding in the World, 541 -550, (2013)

[7] Köhler, T.,Raab, M.,Regensburg, A.,Bergmann, J. P. "Liquid interlayer formation during torsional ultrasonic welding of EN CW004A and EN AW1050"

[8] Hess, A.,Schuster, R.,Heider, A.,Weber, R.,Graf, T. "Continuous Wave Laser Welding of Copper with Combined Beams at Wavelengths of 1030nm and of 515nm", Physics Procedia, 88 - 94, (2011)

[9] Bartl, J.,Branek, M. "Optical and radiating properties of aluminum", Measurement of Physical Review, (2004)

[10] Konuk, A. R., Aarts, R., Huis in 't Veld, B., Sibillano, T., Rizzi, D., Ancona, A. " Closed loop control of laser welding using an optical spectroscopic sensor for Nd: YAG and CO2 lasers ", Proc. ICALEO, Paper 203, Orlando, (2011)

[11] Pascal, S.,Plapper, P. "Spectroscopic studies of dissimilar Al-Cu welding", LTCC, (2018)

[12] LIBS Info: Element Analysis <http://libs-info.com/element_anal/> (21.05.2019)

[13] Semrock "SemrockFilterDetails" < https://www.ahf.de/en/products/spectral-analysisphotonic/optical-filters/individual-filters/bandpass-filters/400-499-nm/2033/485/20brightline-hc > (21.05.2019)

[14] Siemens " Silicon Photodiode for the visible spectral range " <https://cdnreichelt.de/documents/datenblatt/A500/DATASHEET.pdf> (15.05.2019) 